\title{
RAPPORTS HÉTÉROSEXUELS OCCASIONNELS NON PROTÉGÉS DES JEUNES HOMMES LATINO-AMÉRICAINS EN SUISSE : AU-DELÀ DE LA PRISE DE RISQUE
}

\author{
Myrian Carbajal, Lydia Ward et Silvia Schönenberger
}

Presses de Sciences Po | «Agora débats/jeunesses »

2020/1 N $84 \mid$ pages 7 à 21

ISSN 1268-5666

ISBN 978272466253

Article disponible en ligne à l'adresse :

https://www.cairn.info/revue-agora-debats-jeunesses-2020-1-page-7.htm

Distribution électronique Cairn.info pour Presses de Sciences Po.

(C) Presses de Sciences Po. Tous droits réservés pour tous pays.

La reproduction ou représentation de cet article, notamment par photocopie, n'est autorisée que dans les limites des conditions générales d'utilisation du site ou, le cas échéant, des conditions générales de la licence souscrite par votre établissement. Toute autre reproduction ou représentation, en tout ou partie, sous quelque forme et de quelque manière que ce soit, est interdite sauf accord préalable et écrit de l'éditeur, en dehors des cas prévus par la législation en vigueur en France. Il est précisé que son stockage dans une base de données est également interdit. 


\section{Rapports hétérosexuels occasionnels non protégés des jeunes hommes latino-américains en Suisse : au-delà de la prise de risque}

Myrian Carbajal, Lydia Ward, Silvia Schönenberger

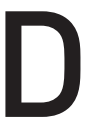

ans les pays occidentaux, l'utilisation du préservatif comme moyen de prévention lors de situations à risque lavec de nouveaux et multiples partenaires) a gagné en importance à la suite de l'irruption de l'épidémie du virus de l'immunodéficience humaine (VIH) au milieu des années 1980. En France, en Allemagne ou encore en Suisse, elle est fréquente lors des premiers rapports sexuels (Beltzer, Bajos, 2008 ; Hessling, 2010 ; Archimi et al., 2016). L'adhésion à la norme de la « bonne contraception », qui implique l'utilisation du préservatif dans les premiers temps, complétée par la pilule, puis l'arrêt du préservatif lorsque les deux partenaires ont fait un test de dépistage $\mathrm{VIH}$, tout en continuant la pilule, fait partie en effet des codes d'entrée dans la sexualité (Bajos, Ferrand, 2006). On pourrait donc penser que l'usage du préservatif, lors des rapports sexuels occasionnels, est de règle entre les partenaires. Cela n'est pourtant pas toujours le cas.

Même si les jeunes qui prennent systématiquement des risques sexuels (Maillochon, 2012) sont rares, le phénomène n'est pas à négliger pour autant. D'ailleurs, comme le souligne Patrick Peretti-Watel (2010), la définition d'une situation à risque par les jeunes qui s'engagent ou qui se sont engagé.e.s dans de telles situations peut être en décalage avec celle donnée « de l'extérieur » par les auteur.trice.s des messages de prévention. PerettiWatel (ibid.) nous invite à considérer les jeunes non pas comme des individus irrationnels et désinvoltes face au risque mais du point de vue de leur rationalité et de leur capacité à fournir des justifications élaborées tendant à affirmer l'innocuité de leurs comportements. Dans le cadre de cet article, nous allons nous intéresser aux rapports hétérosexuels occasionnels, non protégés, de jeunes hommes vivant en Suisse et issus de la migration latinoaméricaine, avec de nouvelles partenaires, dont les risques sont les infections sexuellement transmissibles (IST), comme par exemple le VIH/sida et, 
Rapports hétérosexuels occasionnels non protégés des jeunes hommes latino-américains en Suisse...

dans certains cas, des grossesses non désirées (GND). Plus précisément, notre enquête porte principalement sur les jeunes venant du Pérou, de la Colombie, de la Bolivie et de l'Équateur. Afin de catégoriser les jeunes latinoaméricain·e.s, nous nous sommes basées sur l'origine nationale des jeunes ou de leurs parents. Nous avons privilégié ces quatre pays en raison de leurs points commun en ce qui concerne la contraception laccès parfois limité et/ ou difficilel et de leur législation restrictive en matière d'avortement ${ }^{1}$.

En augmentation constante, la migration latino-américaine en Suisse est passée de 31031 personnes en 2004 à 45071 en $2017^{2}$. Si plusieurs recherches ont déjà étudié différentes facettes et conditions de vie des migrant.e.s latino-américain·e.s arrivé.e.s en Suisse dans les années 1970, 1980 et 1990 (Bolzman, 1996 ; Bolzman et al., 2007), rares sont celles qui se sont penchées sur leur descendance (Carbajal, Ljuslin, 2010 ; Leuenberger, 2007) et encore moins sur le thème de la sexualité. Notre étude, qui vient combler cette lacune, considère la sexualité comme un construit social. John H. Gagnon et William Simon (1973) ont démontré que les comportements sexuels des individus dépendent des apprentissages sociaux, transmis par la socialisation. De plus, ceux-ci participent à la construction de scripts sexuels qui servent de points de référence dans les interactions sexuelles. Le genre traverse les scripts sexuels en tant que «matrice de dispositions générales, comme cadre de socialisation différenciée à la sexualité, et comme guide instruisant » (Monteil, 2016). Ces prescriptions reflètent ce que Judith Butler (1990) nomme « hétéronormativité ».

Selon Butler, l'hétéronormativité est un système de représentations binaires, où le genre masculin concorde avec le sexe biologique masculin et le genre féminin avec le sexe biologique féminin, et où l'hétérosexualité est obligatoire (Rubin, 2012). Dans ce système, les sexualités apparaissent comme asymétriques : la sexualité masculine est caractérisée par l'assertivité, la performance sexuelle, la virilité et un désir sexuel associé aux besoins physiologiques, alors que la sexualité féminine est d'ordre relationnel, liée à l'affectivité et à la conjugalité (Bajos et al., 2008). Gabrielle Richard (2018) souligne combien il est complexe de démontrer le caractère construit des féminités et des masculinités ainsi que de l'hétérosexualité : les «perspectives essentialistes suggérant que la naturalité de l'hétérosexualité et de la complémentarité des sexes se donne à voir dans l'existence de différences innées entre les sexes ou dans les fonctions biologiques associées à la procréation » (Richard, 2018, p. 7).

1. Guttmacher Institute, 2016 /www.guttmacher.org/fact-sheet/facts-abortion-latin-america-andcaribbean).

2. Office fédéral de la statistique (OFS), 2018, Population résidante permanente étrangère selon la nationalité, 1980-2018, tableau : je-f-01.05.01.01 (www.bfs.admin.ch/asset/fr/je-f-01.05.01.01). 
Les travaux qui articulent genre, sexualité et migration, comme par exemple ceux de Christelle Hamel (2002) et d'Isabelle Clair (2008), montrent que la sexualité, comme d'autres pratiques sociales, peut être comprise comme un espace où les rapports de genre se matérialisent et se reproduisent. Selon Clair (2008), « l'ordre du genre », qui repose sur un système hétéronormatif (Butler, 1990), renvoie à la fois à la « mise en ordre (le classement) hiérarchique des groupes de sexe et le rappel à l'ordre des normes de masculinité et de féminité » (Clair, 2013, p. 113). En d'autres termes, le genre, entendu principalement comme "la différentiation et la hiérarchisation des sexes » (Clair, 2012, p. 13) sert d'élément de distinction entre les sexualités. Ces études s'éloignent d'une vision qui voudrait que toute explication concernant les comportements sexuels des jeunes devrait être faite à partir de la « culture d'origine ». Elles soulignent l'importance de ne pas réduire ces jeunes à une seule de ces appartenances et de considérer le contexte socioéconomique, culturel et religieux (Lagrange, Lhomond, 1997) dans lequel ces jeunes interagissent. Comme l'affirme Marta Maia (2009, p. 237), « ce n'est donc pas tant la culture d'origine qui détermine les pratiques et les valeurs des jeunes, mais plutôt le milieu social et institutionnel où ils s'inscrivent. La sociabilité entre pairs qui en est issue consolide alors les manières de faire, de dire et de penser $\gg$.

Notre analyse montre que ces jeunes hommes sont conscients des risques sexuels et qu'ils les contournent par la mise en place d'une stratégie de «tri » de leurs partenaires. La non-utilisation de préservatif relève d'un processus impliquant, dans un premier temps, une évaluation de leur partenaire afin d'écarter, d'une part, les risques d'IST et d'estimer, d'autre part, la responsabilité contraceptive de cette dernière afin d'éviter des GND. Dans un second temps, ces jeunes choisissent de s'en remettre à la décision des femmes en leur laissant le « dernier mot » de la protection ${ }^{3}$. Ce faisant, nos résultats soulignent que les comportements sexuels à risque de ces jeunes hommes s'inscrivent dans un système hétéronormatif (Butler, 1990) où prime le plaisir masculin.

\section{DÉTERMINER L'ABSENCE DU RISQUE SEXUEL CHEZ LA PARTENAIRE}

De manière générale, les jeunes hommes interviewés se montrent réticents à utiliser un préservatif. Ils y voient plusieurs désavantages pratiques, comme le fait de ne pas en avoir lorsqu'ils en ont besoin, d'en posséder, mais en mauvais état, de devoir aller en chercher un, etc. Par ailleurs, l'utilisation du préservatif est vue comme diminuant le plaisir. L'exemple de Marco ${ }^{4}$ est caractéristique d'une logique récurrente chez plusieurs jeunes hommes

3. Lorsque nous parlons de protection, nous faisons référence à l'utilisation du préservatif.

4. Afin de préserver l'anonymat des enquêtés, nous avons choisi des noms d'emprunt. 


\section{Méthodologie}

La présente contribution se base sur une étude qualitative, à caractère exploratoire ${ }^{a}$, qui articule trois thématiques : genre, sexualité et migration. L'étude avait pour objectif de comprendre les représentations sociales et les pratiques sexuelles des jeunes hommes et jeunes femmes âgé·e.s de 16 à 25 ans d'origine latino-américaine. Il s'agissait d'étudier, dans le cadre de leurs expériences hétérosexuelles, leurs pratiques sexuelles en ce qui concerne la protection/contraception et les prises de risques afin d'analyser les rapports entre les sexes, les circonstances ou facteurs qui font qu'ils/elles se retrouvent dans de telles situations et le rôle des représentations sociales dans ces pratiques.

Le plan de recherche a concerné trois groupes principaux distincts selon leur parcours migratoire. Un groupe 1 : des jeunes latino-américain·e.s récemment arrivé.e.s en Suisse, dans un intervalle de un à cinq ans layant suivi la scolarité obligatoire dans leur pays d'originel ; un groupe 2 : des jeunes issu.e.s de la deuxième génération larrivé.e.s entre l'âge de 5 à 13 ans, ayant été scolarisé.e.s en Suisse) ; un groupe 3 : des jeunes latino-suisses, descendant.e.s de couples binationaux. 53 entretiens individuels $(26$ femmes et 27 hommes) et 10 focus groupes ( 5 avec des femmes et 5 avec des hommes, composés en moyenne de 4 participant.e.s), réunissant au total 36 personnes (21 femmes et 15 hommes), ont été réalisés en Suisse Romande (cantons de Fribourg, Vaud et Genève) et en Suisse allemande (cantons de Berne et de Zurich). Les jeunes ont été recrutés par différents canaux : le groupe de professionnel-le.s partenaires de la recherche, la participation à des activités d'associations de migrant.e.s, nos contacts personnels, le bouche-à-oreille, etc. La durée moyenne des entretiens individuels était de 58 minutes (variant de 30 minutes à 2 heures 28 minutes) et celle des entretiens collectifs oscillait entre 50 minutes et 1 heure 45 . Les entretiens individuels et collectifs ont été réalisés dans la langue et les lieux (neutres) choisis par les intervieweuses ou dans ceux proposés par les jeunes.

L'analyse présentée dans cet article se base sur les discours des 18 jeunes hommes qui se sont exprimés sur leurs rapports sexuels occasionnels. Indépendamment de leur parcours migratoire ou de leur niveau de formation, force est de constater que, pour les jeunes hommes, le fait d'évoquer ce type de rapports sexuels lque ce soit du point de vue de leurs pratiques

a. Financée par le Fonds national suisse de la recherche scientifique (FNS) et le fonds stratégique de la Haute école spécialisée de Suisse occidentale (HES-SO), cette étude a été menée dans le cadre de la Haute école de travail social de Fribourg (HETS-FR), en partenariat avec le Forum suisse pour l'étude des migrations et de la population de l'université de Neuchâtel. Elle a été réalisée entre 2012 et 2015 par une équipe de recherche dirigée par Myrian Carbajal et Denise Efionayi-Mäder (voir le site www.hets-fr.ch/fr/recherche/projets/projets-termines). Un groupe de professionnel-le.s représentant les institutions suivantes, que nous remercions pour leur engagement, a accompagné la démarche empirique : l'unité des populations vulnérables de la policlinique médicale universitaire de Lausanne; PROFA Vaud à Lausanne ; les hôpitaux universitaires de Genève ; Santé sexuelle suisse à Lausanne ; le service de planning familial et d'information sexuelle de Fribourg ; l'association Depapaya.org à Genève ; l'association culturelle Matices Peruanos à Fribourg ; l'association ALAS à Berne ; l'association Eurotropicana à Zürich. Le projet a été soumis et a obtenu l'accord de la Commission cantonale d'éthique de la recherche sur l'être humain du canton de Vaud (CER-VD). 
ou des stratégies discursives) est plus valorisant que pour les femmes ${ }^{\mathrm{b}}$. Ces conduites sont assimilables au comportement sexuel masculin. Ces jeunes possèdent la nationalité helvétique (latino-suisse) ou un permis de séjour de type $B$ qui se renouvelle chaque année ljeunes issus de la deuxième génération, jeunes récemment arrivés) ${ }^{c}$. Ils ont soit le statut d'étudiants fréquentant des écoles du secondaire supérieur (gymnases, lycées français) ou du degré tertiaire (universités, hautes écoles), soit le statut d'apprentis d. Ils proviennent de différents milieux socio-économiques, mais leurs parents, qui ont tous connu une déqualification professionnelle, exercent souvent des professions non qualifiées, à l'exception des parents suisses qui occupent des postes de salarié.e.s luniversitaires ou possédant une formation professionnelle supérieure). Notons enfin que parmi les jeunes interviewés, seule une partie a affirmé ne pas avoir utilisé de préservatifs de manière systématique lors de leurs rapports sexuels occasionnels.

b. À noter que sur 27 hommes interviewés, $23(85 \%=23 / 27)$ se sont exprimés sur leurs rapports sexuels occasionnels contre 11 femmes $(42 \%=11 / 26)$. Il convient également de souligner l'importance du contexte de socialisation. Les Latino-Suisses sont en effet les plus nombreux et nombreuses à raconter leurs relations occasionnelles $(71 \%=12 / 17)$, suivi.e.s par ceux et celles de la deuxième génération $(66 \%=12 / 18)$, puis par les jeunes qui ont immigré récemment ( $55 \%=10 / 18$ ). La Suisse fait preuve d'une attitude de tolérance sociale vis-à-vis de la sexualité des jeunes, en dispensant des cours d'éducation sexuelle à l'école et en offrant un accès aux services de santé sexuelle, ce qui n'est pas toujours le cas dans certains pays d'Amérique latine (Geldstein et al., 2000).

c. Étant donné leur problématique particulière, cinq jeunes, récemment arrivés, qui ne possédaient pas d'autorisation de séjour n'ont pas été inclus dans l'analyse.

d. En Suisse, après l'école obligatoire, deux voies, basées sur le niveau scolaire, sont possibles : la première, qui concerne deux tiers des jeunes, consiste à suivre une formation professionnelle initiale ou un apprentissage qui se déroule en école ou en alternance (école/entreprise) et qui permet l'obtention d'un certificat fédéral de capacité (CFC). Dans le deuxième cas de figure, les jeunes réalisent un deuxième degré de formation secondaire (gymnase) qui leur permet d'obtenir un diplôme secondaire supérieur (maturité fédérale ou diplôme) leur donnant accès à des formations tertiaires (universités, hautes écoles spécialisées) : cela concerne un tiers des jeunes (Bolzman, Perregaux, 2008).

interrogés : afficher sa préférence pour les rapports sans préservatif en raison de leur côté « naturel » et de sensations augmentées.

"Est-ce que tu préfères sans ou avec préservatif?

- Sans, la différence, je ne pourrais pas la dire en fait, y'a pas vraiment de raisons, je préfère que ça soit plus naturel, ça coupe le feeling parfois, c'est nul, mais il faut [...]. » (Marco, 20 ans, étudiant au gymnase, deuxième génération.)

Plusieurs études ont montré que les représentations, positives ou négatives, des jeunes concernant le préservatif jouent un rôle sur son utilisation ou sa non-utilisation (Camilleri et al., 2015). Dans la mesure où le préservatif, par exemple, est perçu comme difficile à mettre ou comme ayant un rôle dans la perte d'érection, son usage sera négligé (Amsellem-Mainguy 
Rapports hétérosexuels occasionnels non protégés des jeunes hommes latino-américains en Suisse...

et al., 2017). Certains jeunes mettront ainsi en place une série de stratégies pour convaincre leur partenaire de s'en passer ou pour imposer leur nonutilisation (Pearson, 2006 ; Davis et al., 2014 ; Visser, 2004).

La tendance à éviter l'utilisation d'un préservatif lors d'un rapport sexuel occasionnel va alors de pair avec la nécessité d'évaluer la partenaire pour déterminer les risques d'IST, comme le raconte Ismaël :

"As-tu vécu des situations de peur concernant l'éventualité de contracter une IST ?

$-[\ldots]$ oui $[\ldots]$.

- Que s'est-il passé ?

- [...] Pas de protection oui. Moi je préfère si on ne met pas [de préservatif] mais ça dépend suivant la fille qui, c'est peut-être moi qui va demander à la mettre, si je pense qu'il y a un risque [...]. La plupart du temps, c'est quand même la fille qui prend la décision, si elle est d'accord de faire sans je fais sans, si elle veut la mettre, je vais jamais m'imposer.

- Comment on juge si la situation était risquée ou pas?

- Je me dis si elle est jeune ou moins jeune, ça peut me donner une idée de combien de partenaires elle a eus. Si elle est naîve ou pas... Si elle a l'air influençable ou pas... Quelqu'un qui a du caractère, il va plus souvent s'imposer pour mettre un préservatif et sera pas contaminé, donc sera pas porteur de maladie [...]. » (Ismaël, 24 ans, étudiant universitaire, Latino-Suisse.]

Les possibilités d'une infection sont perçues en fonction du nombre supposé de partenaires. En d'autres termes, comme le suggère Ernesto, un parcours sexuel considéré comme débridé est associé aux risques d'IST.

« Avant, j'étais avec des filles que n'avaient pas encore eu de relations sexuelles. Ma logique était la suivante : si elles n'avaient pas encore eu beaucoup de relations, la possibilité [d'avoir contracté des IST] était beaucoup plus basse [...]. » (Ernesto, 23 ans, étudiant universitaire, Latino-Suisse.)

Notons que les jeunes latino-suisses se soucient principalement des risques d'IST. Les propos d'Ismaël, sans faire mention des GND, comptent sur le tempérament affirmé de la partenaire pour avoir exigé le port du préservatif lors des précédentes relations. Appartenant à la deuxième génération, Éric, contrairement à Ismaël, évoque les risques de GND. Pour lui, les propos de sa partenaire quant à l'utilisation de la pilule l'ont rassuré. Le fait d'assumer la responsabilité de la contraception laisse supposer une personnalité féminine fiable. À l'opposé, pour les jeunes hommes récemment arrivés, l'utilisation des moyens de contraception, qu'ils attribuent surtout aux femmes suisses $^{5}$, est souvent associée au libertinage et à la disponibilité sexuelle (Carbajal, 2020) ${ }^{6}$.

5. Si parfois les jeunes interviewés parlent des «femmes suisses », à d'autres moments, ils parlent des femmes « d'ici ». Cette expression désigne des femmes rencontrées en Suisse, sans pour autant que ces dernières aient forcément la nationalité suisse.

6. Cette représentation n'est pas partagée par tous les jeunes. Certains jeunes soulignent la responsabilité contraceptive des femmes « d'ici » et mettent en question son association avec la disponibilité sexuelle (Carbajal, 2020). 
« On était ensemble pendant deux mois, elle était très attachée aussi. Ensuite, elle m'a dit qu'elle prenait la pilule [...]. Après, si elle a l'initiative de dire "ouais je prends la pilule et je préfère sans [préservatif]", que je la connais depuis assez longtemps, et je sais plus ou moins avec qui elle a des rapports ou si elle m'en parle, là, je dirais aussi oui. » (Éric, 25 ans, apprenti, deuxième génération.)

Aux critères concernant le nombre de partenaires, la capacité d'assumer la responsabilité de la protection et/ou la contraception, s'ajoute celui de l'apparence physique. Un corps sale ou une image non soignée sont associés au risque d'IST, comme le souligne Éric.

« [...] elle n'avait pas l'air malade heureusement [...] avec des personnes qui ont l'air malade, on ne voit pas toujours, mais quand on voit, c'est encore pire! Moi de ce côté-là, je suis assez prudent. Si y'a déjà une apparence, il faut faire plus gaffe [...]. » (Éric, 25 ans, apprenti, deuxième génération.)

Les jeunes hommes mobilisent différents critères len fonction du contexte et des interactions avec la partenairel afin de mettre en place une stratégie d'évaluation de la partenaire et une estimation des risques d'IST et/ ou de GND. Cette appréciation vise à déterminer la nécessité d'utiliser un préservatif. Yaëlle Amsellem-Mainguy et al. (2017), suite

Les jeunes hommes mobilisent différents critères (en fonction du contexte et des interactions avec la partenaire) afin de mettre en place une stratégie d'évaluation de la partenaire et une estimation des risques. à leur analyse de la sexualité des jeunes détenu·e.s en France, qualifient de « sérotriage intuitif » la stratégie utilisée par ces derniers et dernières leur permettant de définir si leurs partenaires sont porteur.se.s d'IST. L'estimation du statut sérologique est liée à l'hygiène corporelle (l'apparence physique) et/ou à la réputation (le fait d'avoir plusieurs partenaires). S'il est vrai que ces procédés ne relèvent pas d'une logique de « risque zéro » mais « bien de tentatives de "faire au mieux" » (AmsellemMainguy et al., 2017, p. 139), les auteur.trice.s affirment que la mise en place de ces stratégies dépend également des représentations du risque. Dans le cadre de notre enquête, les IST, à la différence du VIH/sida, se présentent, d'après ces jeunes, comme des maladies guérissables ou exclues du spectre des possibles.

$\mathrm{Si}$, comme nous venons de le voir, la stratégie déployée permet à ces jeunes hommes d'apprécier l'absence ou la quasi-inexistence de risques d'IST chez leur partenaire et/ou d'écarter des risques de GND, ils laissent, par la suite, le « dernier mot » de la protection aux femmes. Ce faisant, ils se déresponsabilisent de la protection.

\section{LAISSER LE « DERNIER MOT » DE LA PROTECTION AUX FEMMES}

Ismaël, en proclamant que « la plupart du temps, c'est quand même la fille qui prend la décision », ou encore Ernesto, en affirmant qu'il « laisse toujours la possibilité ouverte aux femmes », tout comme d'autres jeunes 
Rapports hétérosexuels occasionnels non protégés

des jeunes hommes latino-américains en Suisse...

hommes que nous avons interviewés, attendent des femmes qu'elles veillent à l'utilisation ou non du préservatif. En d'autres termes, lorsqu'ils estiment avoir écarté les risques d'IST ou de GND, ces jeunes hommes laissent le choix définitif de l'utilisation du préservatif à leur partenaire.

« Je m'adapte à ce que l'autre personne veut. [...] Je laisse toujours la possibilité ouverte [aux femmes], qu'elles me disent "je sais me protéger" [...]. » (Ernesto, 23 ans, étudiant universitaire, Latino-Suisse.)

Laisser le « dernier mot » de la protection à leur partenaire veut dire attendre qu'elles expriment, à travers des paroles ou des actes, leur envie ou leur besoin d'utiliser un préservatif, leur silence semblant en revanche légitimer la non-utilisation du préservatif.

Laisser le « dernier mot » de la protection à leur partenaire veut dire attendre qu'elles expriment, à travers des paroles ou des actes, leur envie ou leur besoin d'utiliser un préservatif, leur silence semblant en revanche légitimer la non-utilisation du préservatif.

Hugo raconte une expérience sexuelle non protégée avec une jeune femme lors d'une soirée festive. Il admet avoir été ivre et ses propos apparaissent un peu contradictoires. En premier lieu, il indique que sa partenaire occasionnelle, face à l'absence de préservatif, l'a rassuré en lui disant que son utilisation n'était pas importante. Mais, dans un second temps, il laisse entendre qu'elle n'a pas tenu de tels propos. L'absence de mention, voire de demande, de la part de sa partenaire concernant l'usage du préservatif, est interprétée par Hugo comme un non-besoin de protection.

« J'étais bourré, c'était dans une fête, la fille, j'allais plus jamais la revoir, j'avais envie, elle avait envie et je n'avais pas de préservatif. Sous l'effet de l'alcool et de l'envie sexuelle, quand t'as 17 ans... Maintenant, aujourd'hui, je ne le ferais pas, parce que j'ai tellement eu peur d'avoir ça [...]. Je n'en avais pas sur moi, on a commencé à s'embrasser. J'en ai pas, merde, qu'est-ce que je vais faire ? On va faire au feeling. Elle me disait que c'était normal quoi, de faire sans préservatif avec tout le monde, enfin je ne sais pas si c'était ça, mais le fait qu'elle m'a rien dit... T'as pas de préservatif... Mais peut être que ce n'était pas le cas, qu'elle était comme moi ! » (Hugo, 20 ans, étudiant au gymnase, deuxième génération.)

Hugo reconnaît toutefois que le silence de la jeune femme aurait pu également signifier un stress de sa part et pas nécessairement son accord pour un rapport sexuel sans protection. Richard de Visser (2004), dans une perspective qualitative, s'est intéressé à l'application tardive du préservatif ${ }^{7}$ et au retrait lors des relations hétérosexuelles des jeunes hommes et femmes (1825 ans) à Melbourne (Australie). Dans ce cadre, il souligne que les femmes doivent gérer un difficile équilibre entre laisser ouverte l'option de ne pas

7. C'est-à-dire au moment de l'éjaculation ou un moment après la pénétration. 
avoir de rapports sexuels ${ }^{8}$ et s'assurer qu'un préservatif soit utilisé en cas de rapport sexuel. Les hommes, quant à eux, ne perçoivent pas cette tension et considèrent que leur partenaire n'a qu'à exprimer leur souhait d'utiliser un préservatif. Pourtant, comme le démontrent les propos d'Hugo, certains jeunes hommes semblent interpréter de manière plus nuancée les non-dits, remettant en doute l'association du silence avec des rapports sexuels non protégés assumés.

Contrairement aux jeunes latino-suisses de la deuxième génération qui laissent, en général, à leur partenaire la responsabilité de la protection sans se poser de questions sur la contraception, les jeunes hommes récemment arrivés, semblent préoccupés par la possibilité de devenir père à un jeune âge. Ayant vécu leurs premières expériences dans un pays où l'utilisation de la pilule par les jeunes femmes n'était pas répandue ils ont dû par exemple utiliser le retrait ou être plus attentifs à l'utilisation du préservatif9. Pour eux, savoir si la partenaire utilise une contraception est important. Elvis souligne toutefois la difficulté, dans le cadre d'un rapport occasionnel, d'interroger sa partenaire sur la prise de la pilule de peur de la réaction de celle-ci qui peut se sentir jugée dans son comportement sexuel.

«Des fois, je parle, on est en train de parler, et moi je ne sais pas que ce jour-là je vais avoir des rapports ; ça arrive comme ça. Peut-être en embrassant, comme ça, je vais même ne pas poser la question. On peut pas prévenir comme ça, je ne peux pas arriver d'un coup : "Ouais, tu prends la pilule?" Je ne peux pas, ça ne le fait pas, mais c'est important de le dire. Mais, si je le dis comme ça, la fille me dira : "Tu me prends pour qui toi ? Tu me connais à peine et tu me poses ça ?" Je pense qu'elle me répondrait comme ça [...]. » (Elvis, 20 ans, apprenti, récemment arrivé.)

Nicolas évoque quant à lui la manière dont, dans des relations occasionnelles avec une femme suisse, la parole de cette dernière a fait foi lors de la négociation de la question de la protection. Le fait qu'elle ait annoncé qu'elle prenait la pilule, associé à son propre désir sexuel, était suffisant, à ses yeux, pour faire confiance.

« Les autres fois étaient sans protection, c'est-à-dire je faisais confiance au fait qu'elle prenait la pilule. [...] C'était quelque chose de passager.

- Comment était négociée la protection?

- Elle m'a dit : "Il n'y a pas de problème, cela fait longtemps que je prends la pilule..." Et comme homme, on fait simplement confiance, et il y a aussi l'envie ; c'est cela qui des fois nous fait commettre des erreurs [...]. » (Nicolas, 23 ans, apprenti, récemment arrivé.)

8. Le fait que les jeunes femmes suggèrent d'utiliser un préservatif serait interprété par les jeunes hommes comme un consentement à avoir des rapports sexuels, alors qu'elles ne veulent pas forcément en avoir.

9. Les jeunes hommes interviewés ont une représentation des femmes suisses ou « d'ici » comme étant majoritaires à utiliser la pilule. Les femmes « latino-américaines », par opposition, sont représentées comme ne sachant pas se protéger et, parfois, comme incapables de s'affirmer visà-vis des hommes (Carbajal, 2020). 
Rapports hétérosexuels occasionnels non protégés

des jeunes hommes latino-américains en Suisse...

Dans les exemples cités ci-avant, le silence des femmes ou, au contraire, la parole de celles-ci pour dire l'absence de risques de GND lutilisation de la pilulel comme la non-formulation du besoin de protection/contraception de la part des hommes semblent faire partie du script des rapports sexuels non protégés. Il semble aller de soi que ce sont les femmes qui doivent aborder le sujet et, si elles ne le font pas, les probabilités que le rapport sexuel se fasse sans préservatif sont grandes.

Lors d'une autre expérience, Hugo, face à l'absence de préservatif, met en évidence que les sentiments qu'il avait pour la fille et le discours de celle-ci sur l'absence de risques lui ont fait choisir la non-protection. On remarque que son discours, qui souligne le caractère «flatteur » des propos de sa partenaire (la grande envie exprimée par celle-ci), est imprégné d'érotisme et de désir sexuel qui l'emportent sur l'importance de se protéger.

«C'était une fille avec qui je flirtais depuis un moment, on n'avait jamais eu l'occasion de se retrouver ensemble, c'était une fille que j'aimais bien, enfin plus que juste comme ça. Elle avait trop envie quoi, elle me disait "viens s'il te plaît"; quand t'entends ça [rires], c'est flatteur ! "Non, mais je n'ai pas de préservatif...", "s'il te plaît, mais c'est bon, j'ai rien...", ça m'a fait des trucs quoi... La meilleure solution ça aurait été de rien faire... mais elle m'a promis, je la connaissais assez bien... C'est aussi une question de confiance, je ne pense pas qu'une personne qui a le sida va prendre le risque de contaminer une personne... ça serait vraiment égoïste quoi. » (Hugo, 20 ans, étudiant au gymnase, deuxième génération.)

À l'instar d'autres études (Amsellem-Mainguy et al., 2017 ; Thomé, 2016), la nôtre souligne que lorsqu'il n'y a pas de préservatif, entre renoncer au rapport sexuel et en avoir un non sans « risque zéro », plusieurs jeunes choisissent la deuxième option.

\section{CONCLUSION}

Notre analyse montre que les comportements des jeunes hommes interviewés, lors de leurs rapports sexuels à risque, s'inscrivent dans le système de l'hétéronormativité (Butler, 1990 ; Rubin, 2012) où la sexualité masculine est associée aux besoins physiologiques et la sexualité féminine à l'affectivité et à la conjugalité (Bajos et al., 2008). En affirmant leur préférence pour l'absence du préservatif, tout en étant conscients de sa nécessité, ils adhèrent, dans un premier temps, à une représentation de la sexualité masculine guidée par le plaisir. Ensuite, la stratégie adoptée pour déterminer l'absence de risques chez leurs partenaires occasionnelles se base sur la capacité de ces dernières à ne pas transgresser ce qui est attendu d'elles dans l'ordre hétéronormatif : une sexualité réservée, cantonnée à l'affectivité et à la responsabilité contraceptive (Bozon, 2012). Finalement, tout en laissant le « dernier mot » de la protection aux femmes et en se montrant virils, ils renforcent l'image de la femme responsable et de l'homme irresponsable en matière contraceptive (Spencer, 1999). En d'autres termes, les femmes, 
vues dans ce contexte comme moralement supérieures aux hommes (Fuller, 1993), sans désir sexuel, mais responsables de la protection du couple, sont censées «freiner » les désirs irrépressibles des hommes (Spencer, 1999).

Cela dit, les jeunes hommes ne sont pas inconscients des risques sexuels d'IST et de GND. Ils expriment leur préférence pour des rapports sexuels sans préservatif, sans pour autant opter automatiquement pour la nonprotection ; ils contournent les risques à travers la mise en place d'une stratégie qui, même si elle n'est pas sûre à $100 \%$, leur permet de se rassurer. En laissant la réponse définitive de la protection aux femmes, ils ne pensent pas se déresponsabiliser ; au contraire, ils voient dans leur acte une prise en considération de l'opinion de la femme et non une imposition de leur volonté. Or, dans les faits, il s'avère difficile d'aller à l'encontre de l'avis de la partenaire lorsqu'elle dit être «sous pilule » ou

Faire preuve de sa masculinité passe par le fait de se montrer à la hauteur des attentes sexuelles vis-à-vis de la sexualité masculine : être fort, sexuellement actif, hétérosexuel et capable de jouir davantage par la non-utilisation du préservatif. au contraire de prendre en compte son avis lorsque celle-ci reste discrète. Si, certains jeunes hommes émettent en effet parfois des doutes sur la manière d'interpréter les non-dits, il n'en demeure pas moins qu'ils ne semblent pas être en mesure de lutter face à l'envie sexuelle (qu'ils sont censés ressentir et démontrer) et face à la perspective d'avoir un rapport dont le plaisir sexuel serait augmenté en raison de la non-utilisation du préservatif. En d'autres termes, les rapports sexuels non protégés s'inscrivent dans un contexte hétéronormatif où l'envie et le plaisir sexuel masculins sont considérés comme dominants. Faire preuve de sa masculinité passe par le fait de se montrer à la hauteur des attentes sexuelles vis-à-vis de la sexualité masculine : être fort, sexuellement actif, hétérosexuel et capable de jouir davantage par la non-utilisation du préservatif. Il ressort ainsi que le fait d'être flexible quant à l'utilisation d'un préservatif maximise le nombre de partenaires, et que le fait d'y renoncer optimise le plaisir sexuel.

Ces résultats montrent, d'une part, l'importance d'encourager des scripts sexuels où les hommes seraient davantage actifs dans les questions de protection et de contraception. Les messages de prévention pourraient inciter ces derniers à davantage de réflexion et favoriser une interrogation sur les non-dits, les gestes, les comportements corporels, etc. D'autre part, il s'agit de questionner autant le contexte hétéronormatif où ces comportements prennent place que le caractère construit des masculinités. Ainsi, les campagnes de prévention pourraient prendre en compte le cadre des références normatives dans lequel les comportements sexuels des jeunes s'inscrivent et les conditions d'intégration socio-économique, politique et culturelle offertes par la société de résidence aux jeunes migrants. Selon Norma Fuller 
Rapports hétérosexuels occasionnels non protégés des jeunes hommes latino-américains en Suisse...

(2001), la masculinité est un processus en tension entre le viril, la sphère domestique et la sphère publique. Dans les situations étudiées, le viril est prépondérant. Pendant la transition vers l'âge adulte, ces jeunes cherchent à devenir autonomes et indépendants vis-à-vis de leur famille et à acquérir des statuts sociaux (travailleur, père, mari, etc.) qui leur permettront de construire leur identité en équilibrant ces trois sphères.

\section{BIBLIOGRAPHIE}

Amsellem-Mainguy Y., Coquard B., Vuattoux A., 2017, Sexualité, amour et normes de genre. Enquête sur la jeunesse incarcérée et son encadrement, Paris, Rapport d'étude de l'INJEP.

Archimi A., Windin B., Delgrande J. M., 2016, « Les rapports sexuels et la contraception des adolescent.e.s en Suisse », Feuille-info HBSC/Addiction Suisse.

Bajos N., Ferrand M., 2006, «L'interruption volontaire de grossesse et la recomposition de la norme procréative $»$, Sociétés contemporaines, $n^{\circ} 61$, p. 91-117.

Bajos N., Ferrand M., Andro A., 2008, « La sexualité à l'épreuve de l'égalité », in Bajos N., Bozon M. (dir.), Enquête sur la sexualité en France. Pratiques, genre et santé, Paris, La Découverte, p. 545-576.

Beltzer N., Bajos N., 2008, « De la contraception à la prévention : les enjeux de la négociation aux différentes étapes des trajectoires affectives et sexuelles », in Bajos N., Bozon M. (dir.), Enquête sur la sexualité en France. Pratiques, genre et santé, Paris, La Découverte, p. 437-460.

Bolzman C., 1996, Sociologie de l'exil. Une approche dynamique. L'exemple des réfugiés chiliens en Suisse, Zurich (Suisse), Seismo.

Bolzman C., Carbajal M., Mainardi G. (dir.), 2007, La Suisse au rythme latino. Dynamiques migratoires des Latino-Américains : logiques d'action, vie quotidienne, pistes d'interventions dans les domaines du social et de la santé, Genève (Suisse), Éditions IES.

Bolzman C., Perregaux C., 2008, « Quelles chances d'accès aux formations professionnelles pour les jeunes étrangers en Suisse ? », in HANHART S. ET AL. (dir.), De la comparaison en éducation. Hommage à Soledad Perez, Paris, L'Harmattan, p. 247-278.

Bozon M., 2012, « Autonomie sexuelle des jeunes et panique morale des adultes. Le garçon sans frein et la fille responsable », Agora débats/jeunesse, $n^{\circ} 60$, p. 121-134. 
Rapports hétérosexuels occasionnels non protégés des jeunes hommes latino-américains en Suisse...

ButLeR J., [1990] 2005, Troubles dans le genre. Le féminisme et la subversion de l'identité, Paris, La Découverte.

Camilleri M., Kohut T., Fischer W., 2015, « Condom use behavioural skills mediate the relationship between condom use motivation and condom use behaviour among young adult heterosexual males : an informationmotivation-behavioural skills analysis », The Canadian Journal of Human Sexuality, n² 2, vol. 24, p. 131-140.

Carbajal M., 2020 [à paraître], « Actitudes y opiniones contrastadas en materia de sexualidad : la construcción de la autonomía individual en el terreno de la sexualidad de las y los jóvenes migrantes latinoamericanas en Suiza », in Galaz C., GISSI N., FACUSE M. (dir.), Migraciones transnacionales : inclusiones diferenciales y posibilidades de reconocimiento, Santiago (Chili), Editorial Universitaria.

Carbajal M., Luuslin N., 2010, « Jeunes sans-papiers d'Amérique latine en Suisse ou devenir adulte sur fond de recomposition de rôles », Lien social et politiques, $n^{\circ} 64$, p. 125-135.

Clair I., 2008, Les jeunes et l'amour dans les cités, Paris, Armand Colin.

CLAIR I., 2012, « Le pédé, la pute et l'ordre hétérosexuel », Agora/débats jeunesses, $n^{\circ}$ 60, p. 67-78.

CLAIR I., 2013, « Pourquoi penser la sexualité pour penser le genre en sociologie ? Retour sur quarante ans de réticences », Cahiers du genre, n०54, p. 93-120.

Davis K. C., Schraufnagel T. J., Kajumulo K. F., Gilmore A. K., Norris J., GEORGE W. H., 2014, «A qualitative examination of men's condom use attitudes and resistance : "It's just part of the game" », Archives of Sexual Behavior, n³, vol. 43, p. 631-643.

Fuller N., 1993, Dilemas de la femineidad. Mujeres de clase media en el Perú, Lima (Pérou), Fondo Editorial, Ponticia Universidad Católica del Perú.

Fuller N., 2001, « No uno, sino muchos rostros. Identidad masculina en el Perú urbano », in Viveros M., Olavarría J., Fuller N. (dir.), Hombres e identidades de género. Investigaciones desde América Latina, Bogota (Colombie), Universidad Nacional de Colombia, p. 266-370.

Gagnon J. H., Simon W., 1973, Sexual Conduct. The Social Sources of Human Sexuality, NewBrunswick (USA)/London (UK), AldineTransaction.

Geldstein R., Infesta Domínguez G., Delpino N., 2000, « La salud reproductiva de las adolescentes frente al espejo : discursos y comportamientos de madres e hijas », in PANTELIDES E. A., BotT S. (dir.), Reproducción, salud y sexualidad en América Latina, Buenos Aires (Argentine)/Genève (Suisse), Biblos/OMS, p. 205-227. 
Rapports hétérosexuels occasionnels non protégés des jeunes hommes latino-américains en Suisse...

Hamel C., 2002, "La "masculinité" dans le contexte de la "galère" : le cas de garçons français maghrébins face aux risques d'infection par le VIH », in Bozon M. ET AL. (dir.), Sida, immigration et inégalités : nouvelles réalités, nouveaux enjeux, Paris, ANRS, p. 85-98.

Hessling A., 2010, Jugendsexualität. Repräsentative Wiederholungsbefragung von 14-bis 17-Jährigen und ihren Eltern - Aktueller Schwerpunkt Migration -, Köln (Allemagne), Bundeszentrale für gesundheitliche Aufklärung (BZgA).

Lagrange H., Lhomond B. (dir.), 1997, L'entrée dans la sexualité. Le comportement des jeunes dans le contexte du sida, Paris, La Découverte.

Leuenberger P., 2007, « Und ständig diese Angst », in NikLaus P.-A., SHÄPPI H. (dir.), Zukunft schwarzarbeit ?, Zürich (Suisse), Édition 8, p. 28-67.

MaiA M., 2009, Sexualités adolescentes, Paris, L'Harmattan.

Maillochon F., 2012, "Premières relations sexuelles et prises de risque. L'éclairage des enquêtes statistiques réalisées en France », Agora débats/ jeunesses, $n^{\circ}$ 60, p. 59-66.

MonteIL L., 2016, « Scripts sexuels », in Rennes J. (dir.), Encyclopédie critique du genre, Paris, La Découverte, p. 584-595.

Pearson J., 2006, « Personal control, self-efficacy in sexual negotiation, and contraceptive risk among adolescents : the role of gender », Sex Roles, n 54, p. 615-625.

Peretti-Watel P., 2010, La société du risque, Paris, La Découverte.

Richard G., 2018, «Perspectives théoriques pour une définition des études sur le genre, les sexualités et les normativités », Revue jeunes et société, $n^{\circ} 1$, vol. 3, p. 4-20.

RubiN G., 2012, « Une conversation avec Gayle Rubin », Raisons politiques, $n^{\circ} 46$, p. 131-173.

SPENCER B., 1999, « La femme sans sexualité et l'homme irresponsable », Actes de la recherche en sciences sociales, $n^{\circ} 128$, p. 29-33.

Tноме́ C., 2016, « D'un objet d'hommes à une responsabilité de femmes. Entre sexualité, santé et genre, analyser la métamorphose du préservatif masculin », Sociétés contemporaines, $n^{\circ} 104$, p. 67-94.

VISSER R. DE, 2004, « Delayed application of condoms, withdrawal and negotiation of safer sex among heterosexual young adults », AIDS Care, $\mathrm{n}^{\circ} 3$, vol. 16, p. 315-322. 


\section{Rapports hétérosexuels occasionnels non protégés des jeunes hommes latino-américains en Suisse...}

\section{LES AUTRICES}

\section{Myrian Carbajal}

Professeure, Haute école de travail social Fribourg, Haute école spécialisée de Suisse occidentale (HES-SO).

Thèmes de recherche : sexualité ; migration ; genre, migration irrégulière et travail domestique; santé sexuelle et reproductive; transitions vers l'âge adulte.

\section{A notamment publié}

CARBAJAL M., LJusLin N., 2012, « Être jeune, privé-e de statut légal et citoyen-ne, est-ce possible? » Tsantsa, no 17, p. 106-115.

CARBAJAL M., LJusLin N., 2015, « L'argent et le travail dans le processus d'autonomisation des jeunes Latino-américain-e-s sans autorisation de séjour en Suisse », Swiss Journal of Sociology/Revue suisse de sociologie, nº 2, vol. 41, p. 223-239.

Carbajal M., Colombo A., Tadorian M., 2019, « Consentir à des expériences sexuelles sans en avoir envie. La logique de redevabilité : responsabilité individuelle ou injonction sociale genrée ? » Journal des anthropologues, n 156-157, p. 197-218.

\section{Lydia Ward}

lydia.wardessagmail.com Chargée de projets au service de la cohésion multiculturelle du canton de Neuchâtel, Suisse.

Thèmes de recherche : l'acquisition des compétences de base pour les personnes issues de la migration et la mise en œuvre de l'Agenda intégration Suisse.

\section{Silvia Schönenberger}

silvia.maria.sch@agmail.com Responsable des projets, Kompetenzzentrum Integration der Stadt Bern (Centre de comptétence d'intégration de la ville de Berne [Suisse]).

Thèmes de recherche : migration ; intégration ; discrimination ; sans-papiers.

\section{A notamment publié}

Mahon P., D'Amato G., Maillefer M., Matthey F., Schönenberger S., Wichmann N., 2013, Mise en œuvre des droits humains en Suisse. Un état des lieux dans le domaine de la migration, Berne, Centre suisse de compétence pour les droits humains (CSDH).

Schönenberger S., Achermann C., 2012, Pilotprojekt «Integrationsvereinbarungen » in Ostermundigen. Schlussbericht der externen Evaluation, Neuenburg, Schweizerisches Forum für Migrations- und Bevölkerungsstudien und Zentrum für Migrationsrecht.

SteIneR I., EfFIOnAYI-Mäder D., SCHÖnenberger S., 2012, Primo-information et protection contre les discriminations : état des lieux en vue de l'élaboration d'un concept vaudois, Neuchâtel, Forum suisse pour l'étude des migrations et de la population. 